\title{
Cryo-TEM study of solid electrolyte interphases in Li-ion batteries
}

Meng Gu

SUSTech, shenzhen, China (People's Republic)

Li-ion batteries are truly changing people's life by innovating the portable electronics and transportations. Pursuit of high-capacity batteries drive us to research intensely for Li-metal anode. However, the problem of Li-dendrites often causes catastrophic failure of batteries. In addition, the atomic structure of solid electrolyte interphase layers (SEI) is largely unknown due to its sensitivity to electron beam, air, and moisture. In order to modify and control the formation and quality of SEI, we need to firstly reveal its native morphology, atomic structure at different discharge and charge states.

Here, we develop an ultra-low dosage technique in the cryo-TEM, which allows us to acquire high resolution cryo-TEM using only $\sim 2-80 \mathrm{e} / \mathrm{A}^{2}$ dosage. The ultralow dosage technique allows us to unveil the native states of SEI during different electrochemical cycling states. As observed in the Li metal anode with DOL-DME LiTFSI electrolyte, the amorphous phases contain tough carbonate polymeric phases, which allows the SEI to swell and shrink with Li metal during cycling. The largely bendable, and flexible properties of the SEI yield long-live batteries with high capacity. We demonstrate the composition of the SEI with EELS, revealing the content of Li, C, O, N, F elements in the SEI. The impurities in Li metal or SEI are resulted from the reactions with electrolyte, salt or additives that are added in the electrolyte to improve the performance of batteries. The impurities inside the Li metal often cause the amorphization of Li metal that could lead to higher resistivity and inactive Li formation during cycling. Statistical analysis reveals that dominant portion of the dead $\mathrm{Li}$ are metallic amorphous $\mathrm{Li}$ rather than the oxidized $\mathrm{Li}$ inside the SEI.

In addition, we track and analyze the fast $\mathrm{Li}$ transportation routes using STEM tomography and wulff construction, disclosing the morpholog of the Li particles during the different cycling states and revealing the dead Li formation mechanism in Li metal batteries. 